\title{
ERRATA: TORSEURS SUR LA DROITE AFFINE
}

\author{
P. GILLE \\ Mathématique, \\ UMR 8628 du C.N.R.S, \\ Université Paris-Sud \\ F-91405 Orsay Cedex, France \\ gille@math.u-psud.fr
}

\begin{abstract}
We clarify two points of our proof of Raghunathan-Ramanathan's theorem [G].
La borne dans la proposition suivante était inexacte dans le cas d'une forme extérieure. La preuve était "canulée" pour la raison suivante: cela n'a aucun sens d'additionner des points d'un appartement d'un immeuble affine, c'est un espace affine. Cette légère modification n'affecte pas le reste de l'article.
\end{abstract}

Proposition 2.3. Soit $\mathfrak{G} / O$ un schéma en groupes semisimples, simplement connexe, tel que $\mathfrak{G}_{K}$ soit un groupe absolument presque $K$-simple de type $\Delta$. Notons $\rho_{d}: H^{1}(\mathcal{G}, \mathfrak{G}(\widetilde{K})) \rightarrow H^{1}\left(\mathcal{G}, \mathfrak{G}\left(\widetilde{K}_{d}\right)\right)$ la restriction induite par l'extension $K_{d} / K$. Si $d_{1}(\Delta) \cdot d_{2}(\Delta) . \sharp \operatorname{Aut}(\Delta)$ divise d, alors

$$
\rho_{d}\left(H^{1}(\mathcal{G}, \mathfrak{G}(\widetilde{K}))\right) \subset \operatorname{Im}\left(H^{1}\left(\mathcal{G}, \mathfrak{G}\left(\widetilde{O}_{d}\right)\right) \rightarrow H^{1}\left(\mathcal{G}, \mathfrak{G}\left(\widetilde{K}_{d}\right)\right)\right)
$$

Il est commode d'isoler le fait suivant.

Lemme 2.3'. Les notations sont celles de la proposition. Soit $\widetilde{\mathcal{A}}$ un appartement de l'immeuble $\widetilde{I}=\mathcal{I}\left(\mathfrak{G}_{\widetilde{K}}\right)$. Soient $x_{1}, \ldots, x_{n}$ des points de $\widetilde{\mathcal{A}}$ de type 0 . Alors le point

$$
\rho_{n}\left(\operatorname{Barycentre}\left(x_{1}, \ldots, x_{n}\right)\right)
$$

est un point de type 0 de l'immeuble $\widetilde{\mathcal{I}}_{n}=\mathcal{I}\left(\mathfrak{G}_{\widetilde{K}_{n}}\right)$.

Démonstration. Soit $T / \widetilde{O}$ le $\widetilde{O}$-tore déployé maximal de $\mathfrak{G}$ défini par l'appartement $\widetilde{A}$. On dispose du diagramme commutatif (*) page 231 de la Section 2.2,

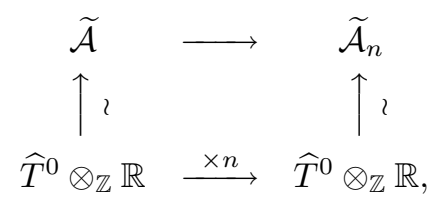

DOI: $10.1007 /$ s00031-005-1010-z

Received April 23, 2005. 
où $\widetilde{\mathcal{A}}_{n}$ désigne l'appartement de $\widetilde{\mathcal{I}}_{n}$ associé au tore $T \times_{\widetilde{O}} \widetilde{O}_{n}$. Les points de $\widetilde{\mathcal{A}}$ de type 0 forment le réseau $\widehat{T}^{0}$ de $\widetilde{\mathcal{A}}$. Pour des sommets $x_{1}, \ldots, x_{n} \in \widehat{T}^{0}$, on a donc

$$
\rho_{n}\left(\text { Barycentre }\left(x_{1}, \ldots, x_{n}\right)\right)=x_{1}+\ldots+x_{n} \in \widehat{T}^{0} \subset \widetilde{A}_{n} .
$$

Ce point est donc un sommet de type 0 de $\widetilde{\mathcal{I}}_{n}$.

Démonstration de la proposition. On pose $d_{1}=d_{1}(\Delta), d_{2}=d_{2}(\Delta), d_{3}=\sharp \operatorname{Aut}(\Delta)$. On peut supposer que $d=d_{1} d_{2} d_{3}$. Rappelons tout d'abord que le schéma en groupes $\mathfrak{G} \times{ }_{O} \widetilde{O}$ est déployé, et ainsi le groupe $\mathfrak{G}_{\widetilde{K}}$ est déployé. Soit $\gamma=[z] \in H^{1}(\mathcal{G}, \mathfrak{G}(\widetilde{K}))$. On considère l'immeuble de Bruhat-Tits $\widetilde{\mathcal{I}}$ (resp. $\widetilde{\mathcal{I}}_{d}$ ) du groupe $\mathfrak{G}_{\widetilde{K}}$ (resp. $\widetilde{\mathcal{I}}_{d}$ ) et la restriction naturelle $\rho_{d}: \widetilde{\mathcal{I}} \rightarrow \widetilde{\mathcal{I}}_{d}$. Ces deux immeubles sont munis de l'action de $\mathcal{G}$, notée $x \mapsto{ }^{s} x(s \in \mathcal{G})$. Le cocycle $z$ induit sur $\widetilde{\mathcal{I}}$ et $\widetilde{\mathcal{I}}_{d}$ une action tordue définie par

$$
x \rightarrow z_{s} \cdot{ }^{s} x \quad(s \in \mathcal{G}),
$$

compatible au morphisme $\rho_{d}$. D'après le théorème de point fixe de Bruhat-Tits ([BrT1, $\S 3.2])$, il existe un point $x$ de $\widetilde{\mathcal{I}}$ fixe par $\mathcal{G}$ pour l'action tordue. Le groupe $\mathcal{G}$ stabilise la facette $F_{x}$ de $\widetilde{\mathcal{I}}$. Si $\mathfrak{G} / O$ est une forme intérieure, tous les sommets de $F_{x}$ sont fixes sous $\mathcal{G}$ et la preuve originale fonctionne avec la borne $d_{1} d_{2}$. Dans le cas général, on doit tenir compte de la $*$-action de $\mathcal{G}$ sur $\Delta$. On note $x_{1}, x_{2}, \ldots, x_{n}$ les sommets de $F_{x}$, ils sont permutés par $\mathcal{G}$ (pour l'action tordue) et appartiennent à un même appartement $\widetilde{\mathcal{A}}$ de $\widetilde{I}$. Quitte à considèrer une sous-facette de $F_{x}$, il est loisible de supposer que $\mathcal{G}$ agit transitivement sur les $x_{i}$. On a $n=\operatorname{Aut}(\Delta) / \operatorname{Aut}(\Delta)_{x_{1}}$, donc $n$ divise $d_{3}$. L'appartement $\widetilde{\mathcal{A}}$ est un espace affine, on peut former le barycentre $x:=\operatorname{Barycentre}\left(x_{1}, \ldots, x_{n}\right)$. Alors $x$ est un point fixe pour l'action tordue de $\mathcal{G}$. Le lemme 2.2 montre que les sommets $\rho_{d_{1} d_{2}}\left(x_{i}\right)$ sont des sommets de type 0 de $\widetilde{\mathcal{I}}_{d_{1} d_{2}}$ appartenant à l'appartement $\rho_{d_{1} d_{2}}(\widetilde{\mathcal{A}})$. Le Lemme $2.3^{\prime}$ appliqué à $\mathfrak{G}_{O_{d_{1} d_{2}}}$ montre que $\rho_{d}(x)=\rho_{d_{3}}\left(\rho_{d_{1} d_{2}}(x)\right)$ est un sommet de type 0 . En d'autres mots, le point $y:=\rho_{d}(x)$ est un sommet de type 0 de $\widetilde{\mathcal{I}}_{d}$ invariant par $\mathcal{G}$. Le reste de la preuve est inchangé. Il existe $g \in \mathfrak{G}\left(K_{d}\right)$ tel que $y=g . c_{\widetilde{\mathcal{I}}_{d}}$. On a $z_{s} \cdot{ }^{s} y=y$ pour tout $s \in \mathcal{G}$, donc si $z_{s}^{\prime}=g^{-1} z_{s}{ }^{s} g$, vu que ${ }^{s} c_{\widetilde{\mathcal{I}}_{d}}=c_{\widetilde{\mathcal{I}}_{d}}$, on obtient

$$
z_{s}^{\prime} \cdot c_{\widetilde{\mathcal{I}}_{d}}=c_{\widetilde{\mathcal{I}}_{d}}(s \in \mathcal{G})
$$

Par suite $z_{s}^{\prime} \in \operatorname{Stab}_{\mathfrak{G}\left(\widetilde{K}_{d}\right)}\left(c_{\widetilde{\mathcal{I}}_{d}}\right)=\mathfrak{G}\left(\widetilde{O}_{d}\right)$ pour tout $s \in \mathcal{G}$. Il résulte que $\rho_{d}([z])=\left[z^{\prime}\right] \in$ $\operatorname{Im}\left(H^{1}\left(\mathcal{G}, \mathfrak{G}\left(\widetilde{O}_{d}\right)\right) \rightarrow H^{1}\left(\mathcal{G}, \mathfrak{G}\left(\widetilde{K}_{d}\right)\right)\right)$.

L'occasion nous est donnée de justifier aussi un point de la démonstration du lemme suivant.

Lemme 3.12. Soit $\gamma \in H^{1}\left(\mathbb{P}_{k}^{1}, G\right)$ tel que ev $v_{\infty}(\gamma)=1$. Alors $\gamma_{/ \mathbb{A}_{k}^{1}}=1$ et $\gamma_{/ \mathbb{P}_{k}^{1} \backslash\{0\}}=1$.

Démonstration. Soit $E / \mathbb{P}_{k}^{1}$ un $G$-torseur représentant la classe $\gamma$. On considère la fibre générique $\gamma_{\eta}=\in H^{1}(k(t), G)$. Si $\gamma_{\eta}$ est anisotrope, le Théorème 3.7 montre que $\gamma=1$ et on a fini. On peut donc supposer la classe $\gamma_{\eta}$ isotrope. Soit $I$ le type d'un sousgroupe parabolique minimal de $E(G)_{k(t)}$. Nous affirmons que $G$ admet un sous-groupe parabolique de type $I$. En effet, soit $X_{I} / k$ la variété des $k$-sous-groupes paraboliques de type $I$ ([SGA3, XXVI.3]). Alors $E\left(X_{I}\right) / \mathbf{P}_{k}^{1}$ est le schéma des $\mathbf{P}_{k}^{1}$-sous-schémas en 
groupes paraboliques de type $I$ du groupe $E(G)$. Celui-ci admet un $k(t)$-point est le critère valuatif de propreté montre que $E\left(X_{I}\right)\left(\mathbf{P}_{k}^{1}\right) \neq \varnothing$. Mais la fibre de $E$ à l'infini est triviale, donc $X_{I}(k) \neq \emptyset$ par spécialisation. Le reste de la preuve est inchangé. Il existe donc un $k$-sous-groupe parabolique $j: Q / k=Z_{G}\left(S_{0}\right) \cdot R_{u} Q \subset G / k\left(\right.$ avec $S_{0} \subset S$ ) tel que $\gamma_{\eta}=j_{*}(\beta) \in \operatorname{Im}\left(H^{1}(k(t), Q)_{a n} \rightarrow H^{1}(k(t), G)\right)$. Alors $E$ admet une réduction à $Q$, i.e., il existe un torseur $F$ sous $Q$ tel que $j_{*} F \stackrel{\sim}{\longrightarrow} E$ et dont la classe de la fibre est $\beta$. Or l'application $G(k) \rightarrow(G / Q)(k)$ est surjective ([BoT, Th. 4.13.a]), d'où l'application $H^{1}(k, Q) \rightarrow H^{1}(k, G)$ un noyau trivial et on a donc $e v_{\infty}(\beta)=1$.

On considère les projections $\pi: Q \rightarrow Q_{\text {red }} \approx Z_{G}\left(S_{0}\right)$ et $p: Z_{G}\left(S_{0}\right) \rightarrow Z_{G}\left(S_{0}\right) / S_{0}$. Alors $p_{*} \pi_{*} F$ est un $\mathbb{P}_{k}^{1}$-torseur sous le groupe semisimple $Z_{G}\left(S_{0}\right) / S_{0}$, dont la fibre à l'infini triviale et dont la fibre générique est anisotrope. Le Théorème 3.6 montre donc que $p_{*} \pi_{*} F$ est isomorphe au torseur trivial. Il résulte que $\left[\pi_{*} F\right]$ provient de $H^{1}\left(\mathbb{P}^{1}, S\right)$. Ceci montre que $\pi_{*} F_{/ \mathbb{A}_{k}^{1}}$ est trivial. Comme $H^{1}\left(\mathbb{A}_{k}^{1}, R_{u} Q\right)=1$, le torseur $F_{/ \mathbb{A}_{k}^{1}}$ (et a fortiori $\left.E_{/ \mathbb{A}_{k}^{1}}\right)$ est trivial. De même, on voit que $E_{/ \mathbb{P}_{k}^{1} \backslash\{0\}}=1$.

Remerciements. Je remercie vivement Vladimir Chernousov de m'avoir signalé ces précisions sur l'article.

\section{Références}

[BoT] A. Borel, J. Tits, Groupes réductifs, Pub. Math. IHES 27 (1965), 55-152.

[BrT1] F. Bruhat, J. Tits, Groupes réductifs sur un corps local I, Publ. Math. IHES 41 (1972), 13-234.

[G] P. Gille, Torseurs sur la droite affine, Transform. Groups 7 (2002), 231-245.

[SGA3] Séminaire de Géométrie algébrique de l'I.H.E.S., 1963-1964, Schémas en groupes, dirigé par M. Demazure et A. Grothendieck, Lecture Notes in Math. 151-153, Springer-Verlag, Berlin, New York (1970). 